\title{
Prediction of Surface Plasmon Resonsnce and More Accurate Representation of Absorption Features Both Below and Above the Bandgap in ZnO Nanorods array - Au Heterostructures
}

Mahla Qaemi

University of Kurdistan

Abdollah Hasanzadeh ( $\square$ mahlaghaemi92@gmail.com )

University of Kurdistan https://orcid.org/0000-0002-7428-9906

\section{Research Article}

Keywords: Zinc oxide nanorods (ZnO NRs) array, Heteronanostructure (HNS), Dispersion model, Charge transfer plasmon, Surface plasmon resonance (SPR), Photogain

Posted Date: November 11th, 2021

DOl: https://doi.org/10.21203/rs.3.rs-855043/v1

License: (c) (i) This work is licensed under a Creative Commons Attribution 4.0 International License.

Read Full License 


\section{Prediction of Surface Plasmon Resonsnce and More Accurate Representation of Absorption Features Both Below and Above the Bandgap in ZnO Nanorods array - Au Heterostructures}

Mahla Ghaemi-moghadam ${ }^{1}$, Abdollah Hasanzadeh ${ }^{1, *}$

1. Department of Physics, Faculty of Science, University of Kurdistan, Sanandaj, Iran

* The corresponding author E- mail: a.hassanzadeh@uok.ac.ir

\section{Abstract}

Well-oriented zinc oxide nanorods ( $\mathrm{ZnO} \mathrm{NRs}$ ) arrays have been grown by low temperature chemical bath deposition on seeded substrates. A gold thin film has obliquely been deposited by DC magnetron sputtering on the ZnO NRs array. The structure, mophology/ chemical identity, vibrational identity have been studied by X-ray diffraction (XRD), field effect- scanning electron microscope/ energy dispersive X-ray spectroscopy (FE-SEM/EDX) and Raman spectroscopy, respectively. The FCC structure of $\mathrm{Au}$ is formed on vertically oriented $\mathrm{ZnO}$ NRsarray. The wavelength dependent photocurrent of $\mathrm{ZnO}$ NRs array- $\mathrm{Au}$ heteronanostructure (HNS) was evaluated by photogain response under red, green and blue laser illuminations. Surface plasmon excitation activates selective response to green laser exposure.

An analytical dispersion formalism has been constructed to fit experimental absorption spectrum over wide spectrum range and to extract precise bandgap energy, subband tailing, dielectric constant and carrier effective mass. The proposed model exploits the Frouhi-Bloomer (FB) parameterization and Gaussian oscillator dispersion to the complex dielectric function for $\mathrm{Au}$ decorated $\mathrm{ZnO}$ NRs array. Sharp variation in the optical absorption around the bandgap edge and the absorption behavior beyond the bandgap edge are covered as well. It is surprising that the surface plasmon resonance 
(SPR) is included without new formalism. The new model has been satisfactorily tested on $\mathrm{CuO}$ optical absorption.

Keywords: Zinc oxide nanorods ( $\mathrm{ZnO} \mathrm{NRs)} \mathrm{array,} \mathrm{Heteronanostructure} \mathrm{(HNS),}$ Dispersion model, Charge transfer plasmon, Surface plasmon resonance (SPR), Photogain

\section{Introduction}

The optical constants of materials are necessary for different applications, such as design, characterization and/or process control of coatings for optics or semiconductor industry and probing into electronic structures [14]. To describe and parameterize the complex refractive index or the dielectric function of semiconductors, various models such as Forouhi and Bloomer (FB) [1], Campi and Coriasso [5], Tauc-Lorentz (TL) [6] and Cody-Lorentz [7] have been developed. To broaden the scope of materials and the spectral range, the models will need more parameters to fit. Recently Franta et al. [3] developed a model which covers a broad spectral range which depends on a large number of parameters.

Optical constants of materials must satisfy some requirements that arise from fundamental properties of the radiation-matter interaction such as causality and linearity. Hence causality results in that the optical constants of a material versus photon energy can be extended with an analytic function to complex energies in the upper half plane, with a given parity and with a fast enough decay towards large energies [8]. The Kramers- 
Kronig (KK) analysis meets all these conditions and connects the imaginary to the real part of the optical constant complex function, and vice-versa. Most presented models optical-constant dispersion of semiconductors are defined with piecewise functions with a change in the functional behavior at the semiconductor bandgap and hence they are not analytic; an exception to this is FB model, even though this model does not work below the bandgap energy. Furthermore, divergences and parity violations are common problems among the mentioned models $[3-4,8]$ One of the well-established and widely used dispersion models to describe the optical properties of the amorphous materials, is the Tauc-Lorentz (TL) model proposed by Jellison and Modine [6] which combine the Tauc expression for $\varepsilon_{2}$ near the band edge with the imaginary part of the complex dielectric function for a single classical Lorenz oscillator $[4,6]$.

Although The TL model severally covers as well smooth variation of band tailing for amorphous material, but for crystalline materials encounters problems due to sharp variations below the bandedge. In addition, discontinuity in the bandgap energy point is an obvious disadvantage of the piecewise functions [2 - 4]. Likhachev et al. [4] presented a modified TL model which leading to a more accurate representation of absorption features below the bandgap energy. A wellknown problem with the application of the TL model to thin films is that it explicitly assumes weak absorption below $\mathrm{E}_{\mathrm{g}}$, i.e., $\varepsilon_{2}(\mathrm{E})=0$ for $\mathrm{E} \leq \mathrm{Eg}$, 
which makes the modeling of the optical spectra of some materials (especially crystalline structures) in the vicinity of the absorption edge problematic [4].

The TL model is not fully analytic and presents mathematical shortcomings. [8]. Rodriguez De-Marcos et al. [8] proposed a procedure that transforms non-analytic TL model into analytic and self-consistent model which used to fit the optical constants of SiC films. They exemplified their model, by imposing the TL dispersion to the Urbach tail, for the optical constants of $\mathrm{Si}_{3} \mathrm{~N}_{4}$.

In TL model the imaginary part of the dielectric constant $\varepsilon 2$ turns zero below the material bandgap energy, whereas in practice, semiconductors and dielectrics are known to experience an exponential decay of the absorption coefficient, $\alpha$, below the bandgap, which is called the Urbach tail. Valuable models were proposed to incorporate the Urbach tail to the TL model by Foldyna et al. [9] and a similar extension to the CodyLorentz model was performed by Ferlauto et al. [10]. However, the corrected models kept using the piecewise functions, so that the lack of analyticity is not solved. Likhacher et al. [4] and Franta et al. [11] reported that, the absorption below the band gap often does not correspond to the Urbach tail and the simple combination of Urbach subband tail with the TL oscillator(s) is insufficient near the band gap. 
Li et al. [2] developed a model for describing the absorption coefficient based on optical transitions with a normalized average dipole matrix element. Their model was employed to fit the optical absorption edges of annealed amorphous $\mathrm{Si}: \mathrm{H}$ films and subsequently to determine the values of the optical gap, tail breadth and mobility gap. The relationship between the optical gap and the band tail breadth reveals that the tail breadth depends linearly on the structural disorder [2]. Li et al. [2] and Franta et al. [3] used piecewise functions to model optical absorption and band parameters that requires various parameters and worry about the continuity of the absorption behavior.

Previous dispersion model were often concentrated on disordered and amorphous materials and glassy system [2, 12, 13]. Few model concentrate on optical constant of crystalline materials, hybrid structure and metal - semiconductor composite. The FB dispersion is very popular to represent the dielectric functions (complex refractive index) of amorphous semiconductors and dielectrics. However, an improved model is required to predict the fundamental bandedge absorption and wide range absorption below and above the band to band transition for multi- component crystalline systems. A Guassian oscillator distribution imposed FB dispersion has incorporated by additional bounded Lorentz oscillators and free carrier absorption with transition energies located below the bandgap. In this paper we propose a model 
which well predicts a meaningful value for the bandgap energy and precise behavior beyond the band to band absorption, subband tailing an surface plasmon resonance (SPR) without individual formalism. An example has been provided to illustrate the practical use of the model.

\subsection{Experimental methods}

\section{1. $1 \mathrm{ZnO}$ NRs- Au array growth}

$\mathrm{ZnO}$ NRs array was grown by chemical bath deposition in an equimolar $25 \mathrm{mM}$ zinc nitrate hexahydrate $\left(\mathrm{Zn}\left(\mathrm{NO}_{3}\right) \cdot 6 \mathrm{H}_{2} \mathrm{O}\right)$ and $25 \mathrm{mM}$ hexamine $\left(\mathrm{C}_{6} \mathrm{H}_{12} \mathrm{~N}_{4}\right.$, HMTA) aqueous solutions on seeded substrates at low temperature of $80{ }^{\circ} C \quad[14,15,16]$. The seed layer was deposited by radiofrequency (RF) magnetron sputtering on $\mathrm{Si}$, quartz, fluorine doped tin oxide (FTO) substrates. The ZnO NRs array was washed with de-ionized (DI) water to remove the surface contaminants and baked at $80^{\circ} \mathrm{C}$. An Au nanosheet was deposited on the $\mathrm{ZnO}$ NRs surface by DC magnetron sputtering at oblique angle $\left(80^{\circ}\right)$.

\subsubsection{Characterization}

The crystalline structure was investigated using $\mathrm{X}$ - ray diffractometry $(\mathrm{XRD}$, Bruker $\mathrm{D} 8$ ADVANCE). The surface morphology and chemical composition were studied by field emission scanning electron microscope and energy dispersive $\mathrm{X}$ ray spectroscopy (FE-SEM/EDX, Hitachi S-4800), respectively. The thickness was determined by cross sectional FE-SEM images, oscillating quartz crystal monitoring and reflectance ellipsometry (LEOI-44 model, wavelength 632.8nm of He- Ne laser). The optical absorption spectra were recorded by an UV-Vis- near IR spectrophotometer (Avantes-Spec2048). The Raman spectrum was measured at room temperature using a micro Raman spectrometer (Taksan, TakRam Model: P50C0R10) with 532 nm line of Nd: YAG laser as an exciting light source. 


\subsection{3 photogain measurement}

The bare $\mathrm{ZnO}$ NRs and ZnO NRs- $\mathrm{Au}$ array heteronanostructure (HNS) were sandwiched between Ag and fluorine doped tin oxide (FTO) electrodes. Current-Voltage characteristic was measured under the red, green and red laser irradiation. A source measurement (model 2450 Keithley Instrument) was used to detect the output signals generated from the HNS.

2.2 Brief review of the dispersion models

The main models related to the topic of the present paper are briefly reviewed.

\subsubsection{Lorentz oscillator dispersion and Drude model}

The Lorentz oscillator model is one of the oldest classical dispersion models for the predicting the dielectric functions of materials, which assumes that the material can be described as a collection of non-interacting dipoles and damped harmonic oscillators, and it is still being used successfully to explain experimental results [4]. The electronic and vibrational dipoles are both examples of bound oscillators [17].

The imaginary part $\varepsilon_{2}$ of the dielectric function is given by $[4,17]$

$$
\varepsilon_{2 L}(E)=\sum_{j} \frac{A_{j} E_{0 j} \Gamma_{j}^{2} E}{\left(E_{0 j}{ }^{2}-E^{2}\right)^{2}+\Gamma_{j}^{2} E^{2}}
$$

where the fitting parameters are the $\mathrm{j}$-th Lorentz oscillator position or natural resonant energy $\left(E_{0 j}\right)$, the amplitude $\left(A_{j}\right)$ and the broadening parameter of the bound electrons $\left(\Gamma_{j}=\hbar \gamma_{j}\right)$ and $\gamma_{j}$ damping rate.

Metals and doped semiconductors contain significant numbers of free electrons. These electrons are not bound to any atoms, and therefore do not experience any restoring forces when they are displaced. Hence the natural resonant energy for free electrons $E_{0}=0$. The free carrier 
contribution to the imaginary part of the dielectric function $\varepsilon_{2 D}$ is given by Drude model $[17,18]$

$\varepsilon_{2 D}(E)=\frac{\varepsilon_{\infty} E_{p}^{2} \Gamma_{D}}{E^{3}+\Gamma_{D}^{2} E}$

\subsubsection{Gaussian oscillator dispersion model}

The atomic bond lengths and angles for amorphous and glassy materials are normally distributed around average values [4, 19] hence, the Gaussian oscillator model may be a good approximation for amorphous and glassy materials. Likhachev et al. [4] first proposed the Guassin Oscillator dispersion may be used for modeling the optical functions of molecular resists or copolymers as well as for the dielectric functions of disordered materials in the infrared range.

A parameterized form for the imaginary part of the dielectric function

$\varepsilon_{2} \mathrm{G}$ is defined by

$\varepsilon_{2 G}(E) \propto \exp \left(-\left(\frac{E-E_{G}}{\sigma}\right)^{2}\right), \quad \sigma=\frac{\Delta E}{2 \sqrt{\ln 2}}$

where $\Delta E$ is the Guassian broadening parameter and $\mathrm{EG}_{\mathrm{G}}$ is the peak position of Guassian oscillator dispersion.

\subsubsection{Forouhi - Bloomer dispersion model}


Easwarakhanthan et al. [20] reported on optical properties of plasma deposited fluorocarbon (FC) films on Si substrates. The obtained optical constants with spectroscopic ellipsometry (SE), were evaluated using Forouhi-Bloomer (FB) and Tauc-Lorentz (TL) dispersion models. Both dispersions models yielded a low refractive index of 1.39 and identical model layer thicknesses. Specifically, the FB dispersion better describes the region near absorption cutoff with taking up a lower optical band gap (OBG) than that of the TL dispersion model. Thereby indicating that particular fluorocarbon film absorptions which are included in the FB dispersion model are excluded in the TL model. Also, the FB index spectrum tends to peak towards a broad maximum in the ultraviolet (UV) wavelength range, whereas this trend is absent in the TL index spectrum. The analytical Forouhi-Bloomer dispersion equations describe the index of refraction $n(E)$ and the extinction coefficient $k(E)$ for a wide variety of semiconductors and dielectrics, produced under a variety of processing conditions. The Forouhi-Bloomer dispersion equations are [1]

$$
\begin{aligned}
& k(E)=\sum_{i=1}^{q} \frac{A_{i}\left(E-E_{g}\right)^{2}}{E^{2}+B_{i} E+C_{i}} \\
& n(E)=n(\infty)+\sum_{i=1}^{q} \frac{B_{0 i} E+C_{0 i}}{E^{2}+B_{i} E+C_{i}}
\end{aligned}
$$

Each term in the summations for $\mathrm{k}(\mathrm{E})$ and $\mathrm{n}(\mathrm{E})$ contributes either a peak or a shoulder to the spectra, respectively. Thus, the number of terms equals the number of discernible spectral peaks and shoulders. In Eq. 1, 
$E_{\mathrm{g}}$ represents the bandgap energy which defined as that value of photon energy that $k(E)$ exhibits an absolute minimum. The parameters $A_{i}, B_{i}$ and $\mathrm{C}_{\mathrm{i}}$ are not mere fitting parameters; they depend on the electronic configuration of the material [1]. The quantities $\mathrm{B}_{0 \mathrm{i}}$ and $\mathrm{C}_{0 \mathrm{i}}$ are not independent parameters, they depend on $A_{i}, B_{i}, C_{i}$ and $E_{g}$. Eqs 1 and 2 are valid over a very wide range of photon energies spanning ultraviolet, visible, and near infrared. Furthermore, they are related through the Kramers-Kronig relation [1].

\subsubsection{Model construction}

The total imaginary part of the dielectric function includes the bound electrons, band to band transition and free carriers contributions. So that

$\varepsilon_{2}(E)=\varepsilon_{2 L}(E)+\varepsilon_{2 F B-G}(E)+\varepsilon_{2 D}(E)$

where the first term is a single Lorentzian dispersion for the bound electrons $[4,17]$

$\varepsilon_{2 L}(E)=\frac{A_{L} E}{\left(E_{0}{ }^{2}-E^{2}\right)^{2}+\Gamma_{b}^{2} E^{2}}$

and the second terms is selected as the product of single FB terms and Gaussians peaks.

$\varepsilon_{2 F B-G}(E)=\varepsilon_{2 F B}(E) * \varepsilon_{2 G}(E)=\frac{A_{F B}\left(E-E_{t h}\right)^{2}}{E^{2}+B E+C} \cdot \exp \left(-\left(\frac{E-E_{G}}{\sigma}\right)^{2}\right)$

$E_{t h}$ is the threshold energy for absorption onset and related to optical bandgap energy and band tailing width $\left(E_{\Delta}\right), E_{t h}=E_{g}-E_{\Delta}$. 
The absorption coefficient is related to the extinction coefficient and imaginary part of the dielectric function, $\alpha=\frac{2 k \omega}{c}$ and $k=\frac{\varepsilon_{2}}{2 n(\infty)}$. The root of the second derivative of the absorption coefficient in the shoulder interval is selected as the bandgap energy

$\left.\frac{d^{2} \alpha}{d E 2}\right|_{E=E g}=0$

The real part of the dielectric function $\varepsilon_{1}$ for the Lorentz, Forouhi- Bloomer Gaussian and Drude oscillator models is obtained by the Kramers-Kronig integration [17] of the $\varepsilon_{2}$ functions described by Eqs. (2), (7) and (8):

$\varepsilon_{1}(E)=\varepsilon_{\infty}+\frac{2}{\pi} P \int_{E_{g}}^{\infty} \frac{\xi \varepsilon_{2}(\xi)}{\xi^{2}-E^{2}} d \xi$

where $\varepsilon_{\infty}$ represents the value of the real part of the dielectric function $\varepsilon$ at infinite energy and it is an additional fitting parameter in the oscillator model, the P stands for the Cauchy principal part of the integral.

The total real part of the dielectric function includes

$\varepsilon_{1}(E)=\varepsilon_{1 L}(E)+\varepsilon_{1 F B-G}(E)+\varepsilon_{1 D}(E)$

where the first term is the Kramers-Kronig integration of a single Lorentzian dispersion for the bound electrons [17].

$\varepsilon_{1 L}(E)=\frac{\frac{A_{L}}{\Gamma_{b}}\left(E_{0}{ }^{2}-E^{2}\right)}{\left(E_{0}{ }^{2}-E^{2}\right)^{2}+\Gamma_{b}{ }^{2} E^{2}}$

The second terms in Eq. 11 is the Kramers-Kronig integration of the product of single FB terms and Gaussians peaks $\varepsilon_{2 F B-G}(E)$ which is obtained using residual (Res) calculus. 
$\varepsilon_{1 F B-G}(E)=\frac{2}{\pi} P \int_{0}^{\infty} \frac{\xi \varepsilon_{2 F B-G}(\xi)}{\xi^{2}-E^{2}} d \xi \propto \operatorname{Res}(E)+\operatorname{Res}(-E)$

where

$\operatorname{Res}(u)=\frac{1}{2} \frac{\left(E-E_{t h}\right)^{2} \exp \left(-H(E-E G)^{2}\right)}{E\left(E^{2}-B E+C\right)}$

The third term is the real part of Drude dielctrice function [18]

$\varepsilon_{1 D}(E)=1-\frac{\varepsilon_{\infty} E_{p}^{2}}{E^{2}+\Gamma_{D}^{2}}$

\subsubsection{Refractive index, dielectric constant and effective mass}

The refractive index of the bare $\mathrm{ZnO} \mathrm{NRs}$ array and $\mathrm{ZnO}$ NRs- $\mathrm{Au}$ heterostructure was calculated using the Moss relation [21], which is directly related to the fundamental energy band gap $(\mathrm{Eg})$,

$E_{g} n^{4}=108 e V$

A different relation between the refractive index and the band gap energy was presented by Herve and Vandamme as follows [22, 23],

$n=\sqrt{1+\left(\frac{13.6}{E_{g}+3.4}\right)^{2}}$

Dimitrov - Sakka introduced another relation as follows [24]

$\frac{n^{2}-1}{n^{2}+2}=1-\sqrt{\frac{E_{g}}{20}}$

The high-frequency dielectric constant $\left(\varepsilon_{\infty}\right)$ was calculated from the following relation [25], 
$\varepsilon_{\infty}=n^{2}$

The static dielectric constant $\left(\varepsilon_{s t}\right)$ of the films was calculated using a relation which expresses the energy band gap dependence of $\varepsilon_{s t}$ for semiconductor compounds in the following form [22],

$$
\varepsilon_{s t}=-33.26876+78.61805 E_{g}-45.70795 \mathrm{E}_{\mathrm{g}}^{2}+8.32449 \mathrm{E}_{\mathrm{g}}^{3}
$$

Similar to the static dielectric constant, a relation expressing the energy band gap dependence of the electron effective mass for the semiconductor compounds has the following form [21],

$$
\frac{\mathrm{m}_{\mathrm{e}}^{*}}{\mathrm{~m}_{0}}=5.17004-7.46699 \mathrm{E}_{\mathrm{g}}+3.63268 \mathrm{E}_{\mathrm{g}}^{2}-0.57525 \mathrm{E}_{\mathrm{g}}^{3}
$$

\section{Results and discussions}

\subsection{Morphology and chemical content analysis}

Fig. 1 shows FE-SEM images of the bare ZnO NRs and ZnO NRs - Au array HNS coated with various $\mathrm{Au}$ thickness on seeded $\mathrm{Si}$ substrate. Fig. $1 \mathrm{a}-\mathrm{c}$ shows top views and cross-sectional views of bare $\mathrm{ZnO}$ NRs and $\mathrm{ZnO}$ NRs - Au array with 10 and $25 \mathrm{~nm}$ coated gold nanosheets. The well-aligned NRs array is columnar on seeded Si substrate. The obtained ZnO nanorods show a hexagonal shape and a growth direction perpendicular to the substrate. A pin shape on the top was obtained for NRs synthesized for Au thickness of 40nm. The number density, mean diameter and mean height of the bare $\mathrm{ZnO}$ NRs were $384 \mathrm{rods} /(\mu \mathrm{m})^{2}, 35 \mathrm{~nm}$ and $130 \mathrm{~nm}$, respectively. To analyze the elemental compositions of the bare ZnO NRs array and ZnO NRs- Au array HNS, an EDX spectra were recorded (Fig. 1(d)-(f)). The characteristic peaks of $\mathrm{O}$ appeared at $0.5 \mathrm{keV}$, and the characteristic peaks of $\mathrm{Zn}$ appeared at 1.8 and $8.9 \mathrm{keV}$, further clarify the formation of 
$\mathrm{ZnO}$ NRs array. The appearance of the characteristic peak of $\mathrm{Au}$ at $2.2 \mathrm{keV}$ confirms the $\mathrm{ZnO}$ NRs- Au formation. The size of the dewetted Au NPs and its concentration are tuned by varying the thickness of the gold nanosheet.

(a)

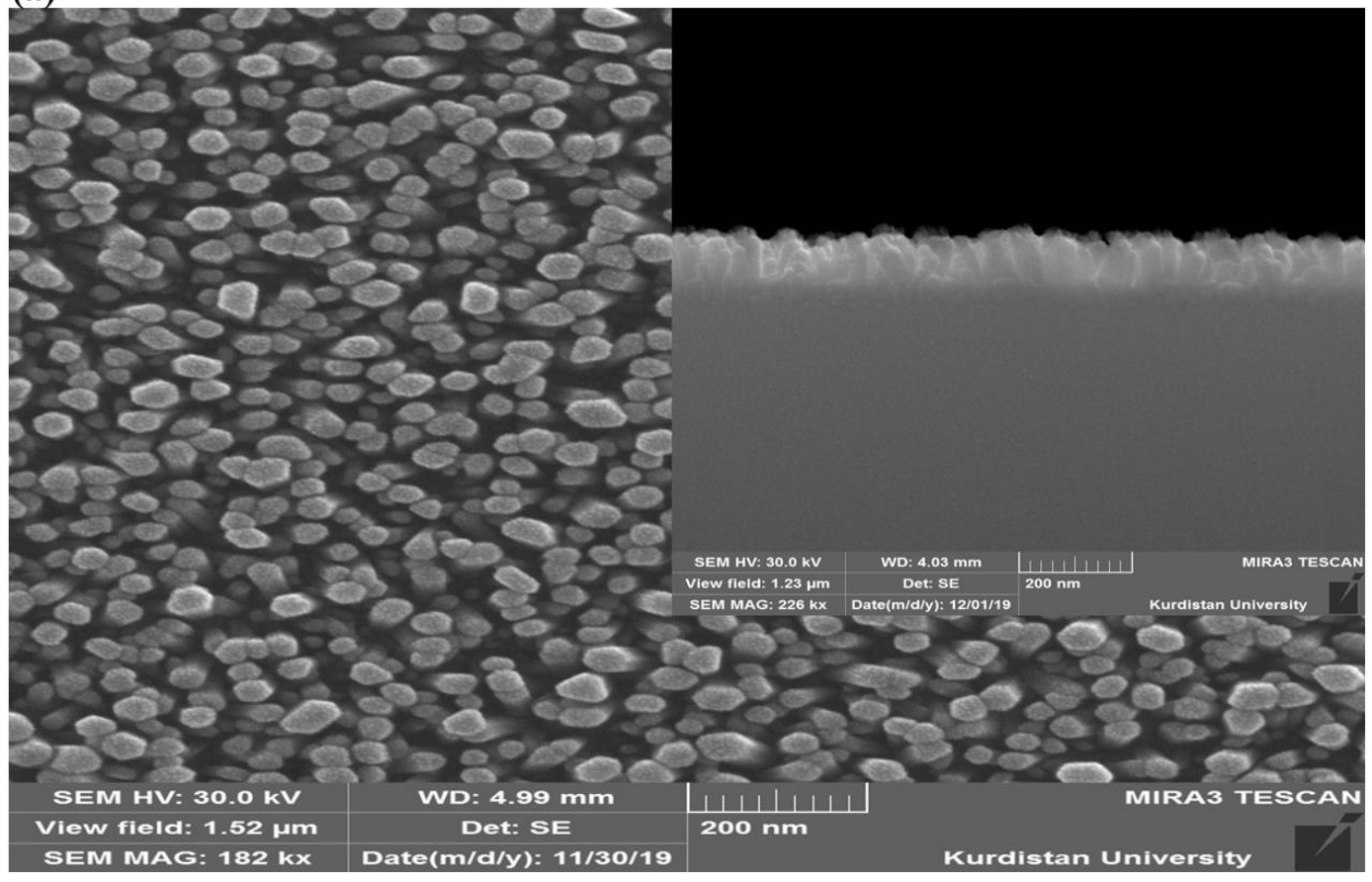

(b)

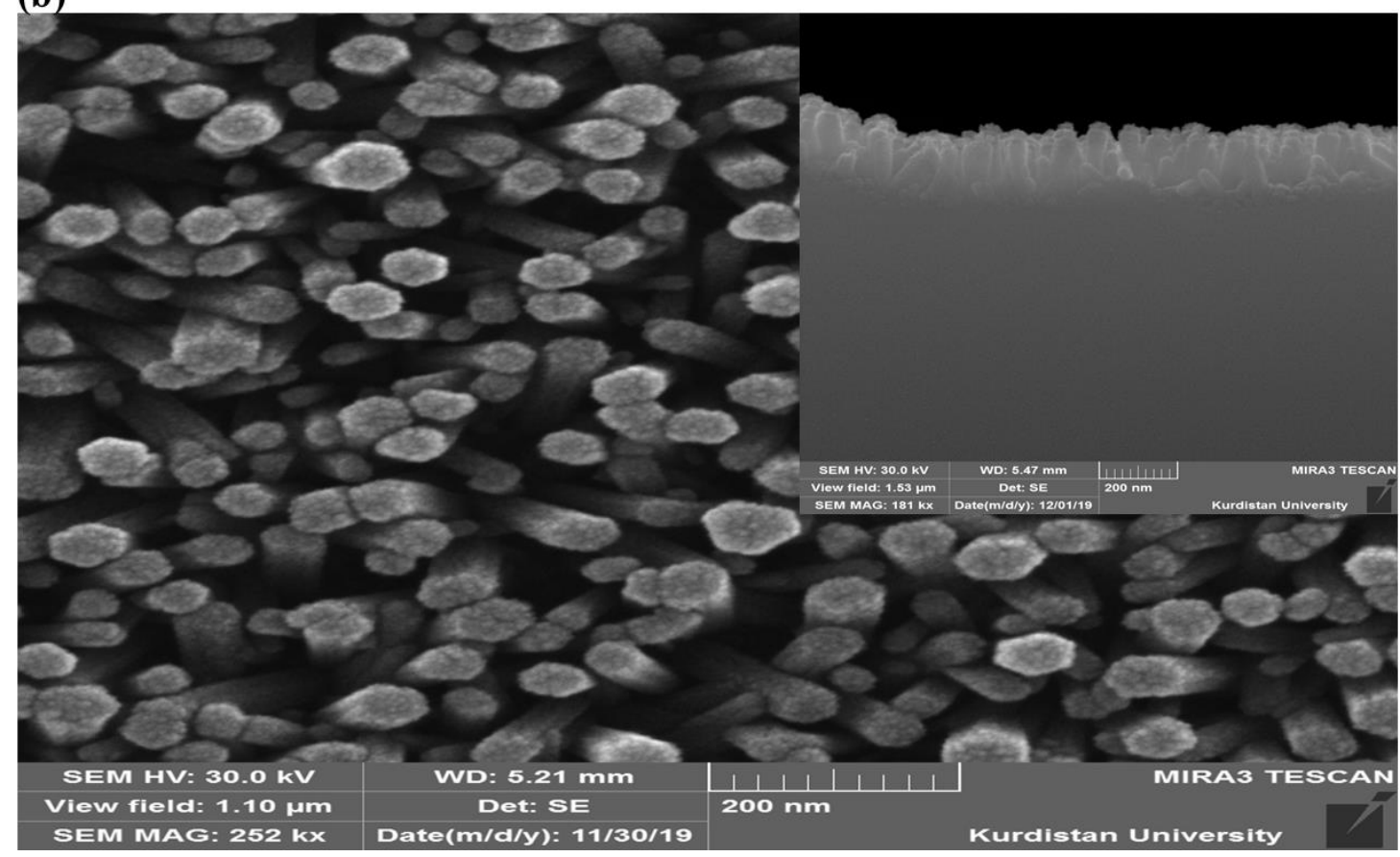


(c)
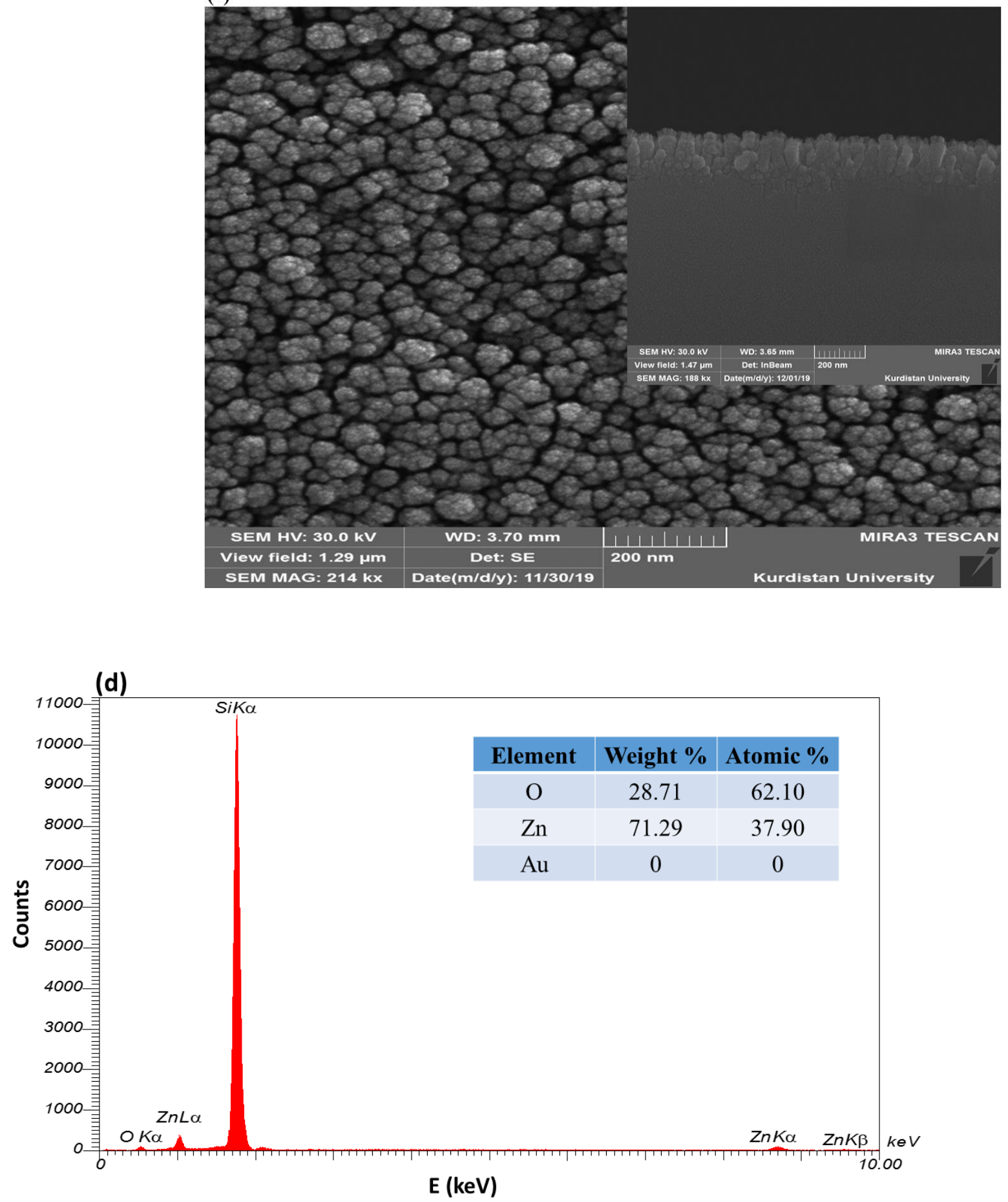

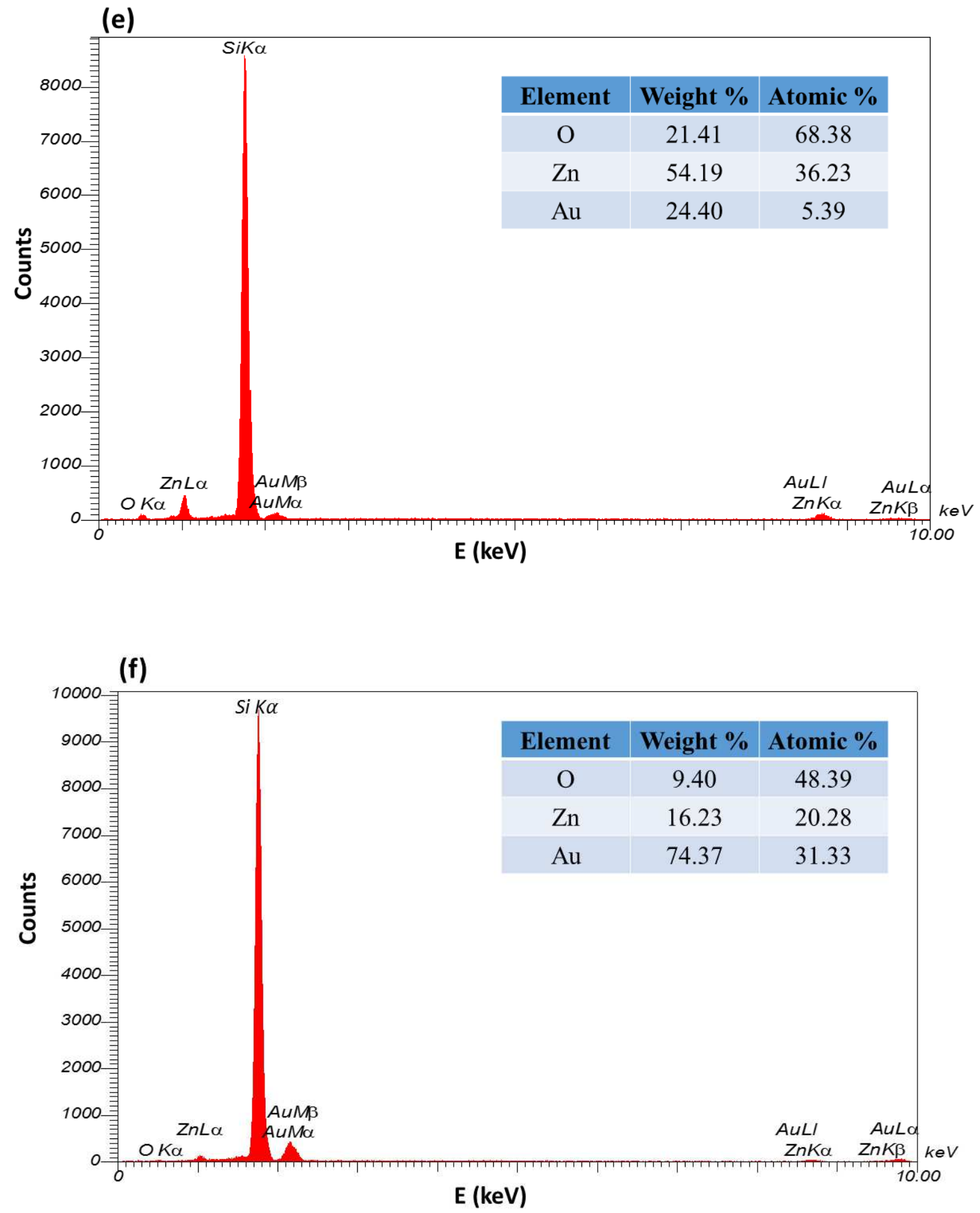

Fig. 1: Top view and cross section FE-SEM images and EDX of (a, d) bare ZnO NRs array (b,e) $10 \mathrm{~nm} \mathrm{Au}(\mathrm{c}, \mathrm{f}) 25 \mathrm{~nm} \mathrm{Au}$

\subsection{Structural determination and lattice dynamics}


Fig. 2 shows the XRD patterns of the bare $\mathrm{ZnO}$ nanorods and $\mathrm{ZnO} \mathrm{NRs}-\mathrm{Au} 25 \mathrm{~nm}$ array on the Si (001) substrate. The diffraction peaks centering around $2 \theta=31.76^{\circ}, 34.44^{\circ}, 36.42^{\circ}, 47.58^{\circ}$, $56.64^{\circ}, 62.9^{\circ}, 66.5^{\circ}, 68.02^{\circ}$, and $69.1^{\circ}$ can be perfectly assigned to reflections of (100), (002), (101), (102), (110), (103), (200), (112), and (201) crystallographic planes, respectively, which well indexed with the single-phase hexagonal wurtzite $\mathrm{ZnO}$ structure (JCPDS 36-1451). The highest intensity for diffraction peak of (002) crystal planes indicates the preferred crystallographic orientation of $\mathrm{ZnO}$ NRs along the (002) direction.

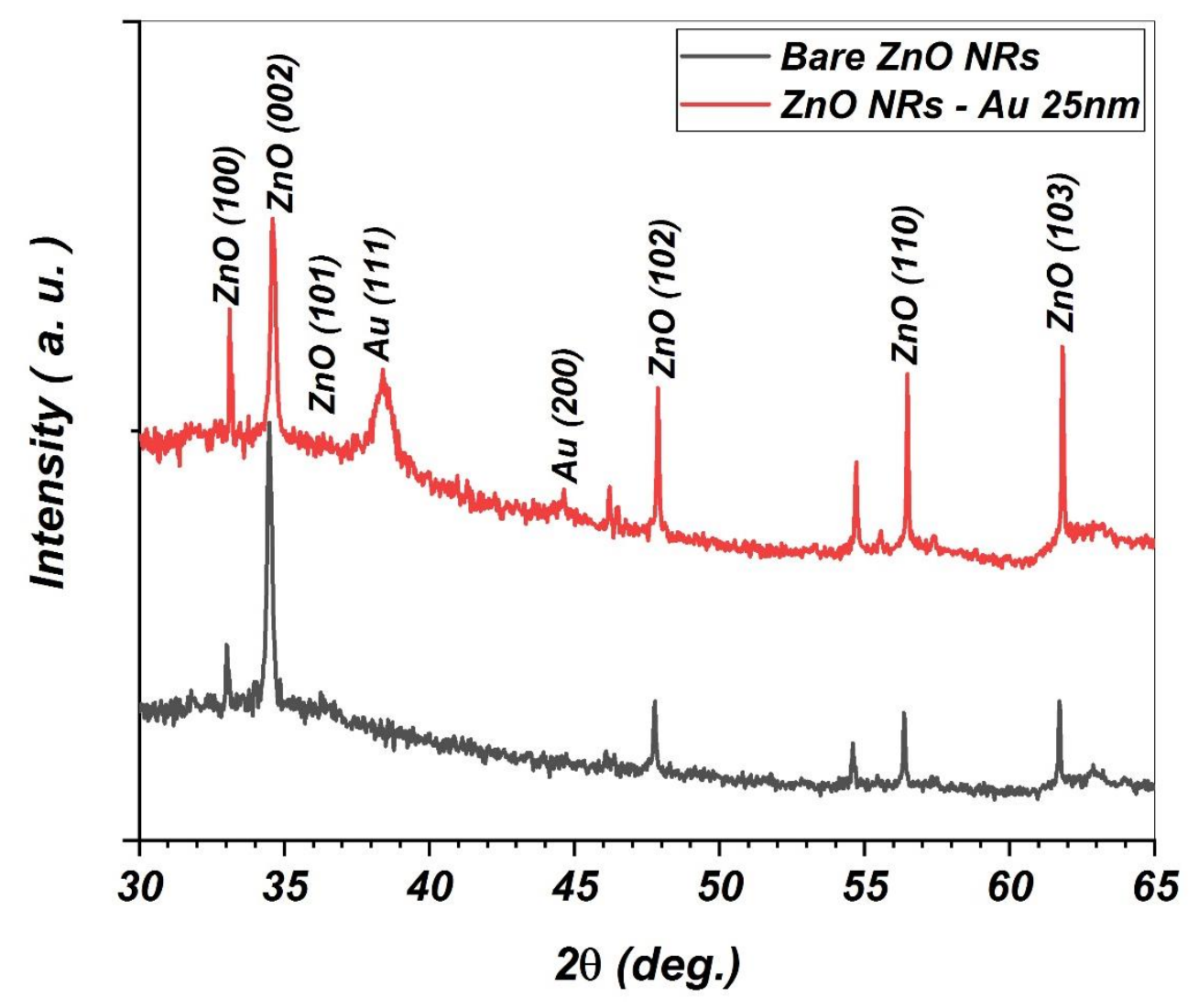

Fig. 2: XRD pattern of the bare $\mathrm{ZnO}$ NRs array and $\mathrm{ZnO}$ NRs-Au $25 \mathrm{~nm}$ array HNS

In addition, three diffraction peaks centering around $2 \theta=38.32^{\circ}$ and $64.92^{\circ}$ depict the reflection of (111) and (220) planes of fcc Au structure (JCPDS no. 00- 001- 1172). Hence, the binary ZnO NRs- Au HNS exhibits good crystalline structures. The XRD results and the EDX data 
simultaneously confirm that the Au incorporated ZnO NRs array. The broad (111) peak shows the nanoscale size of Au nanoparticles.

The peaks between 942 and $1145 \mathrm{~cm}^{-1}$ may be attributed to the second order surface phonon modes. Breakdown of the translation symmetry in the bare $\mathrm{ZnO}$ NRs array cause to the surface phonon modes which highly localized near the boundary of the NRs [16]. Since the surface area is considerably increased due to the fabrication of $\mathrm{ZnO} \mathrm{NRs}$, the interaction of light with the surface should activate the surface phonon modes. This gives rise to two branches of surface modes between the TO and LO phonon frequencies of $\mathrm{ZnO}$ [26]. The frequency of the upper surface phonon mode (UM) is normally below the corresponding bulk $\mathrm{E}_{1}(\mathrm{LO})$ value $\left(591 \mathrm{~cm}^{-1}\right)$ [29]. The lower surface phonon mode (LM) is clearly reported at around $475 \mathrm{~cm}^{-1}$ in the highly c-axis oriented and well-isolated vertically aligned $\mathrm{ZnO}$ NRs [16]. The oxygen deficiency results in a peak at $578 \mathrm{~cm}^{-1}$ that positioned between $\mathrm{A}_{1}(\mathrm{LO})$ and $\mathrm{E}_{1}(\mathrm{LO})$ optical phonon mode $[16,27-$ 28]. The peaks around 287 and $970 \mathrm{~cm}^{-1}$ may be attributed to orders of surface phonon modesor oxygen deficiency. 


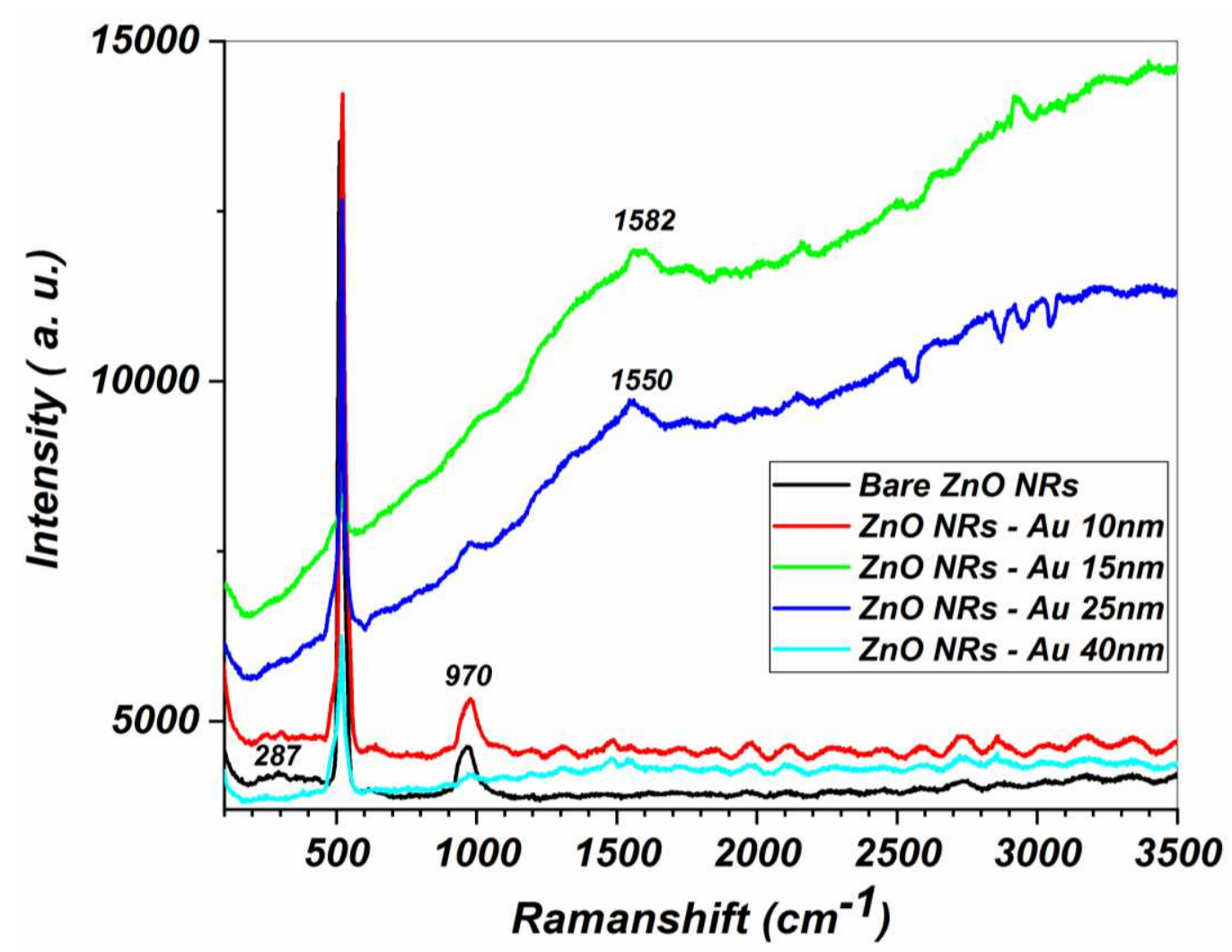

Fig. 3: Raman spectra of bare $\mathrm{ZnO}$ NRs array and $\mathrm{ZnO} \mathrm{NRs}-\mathrm{Au}$ array heterostructure

The peak around $1550-1582 \mathrm{~cm}^{-1}$ for $\mathrm{ZnO}$ NRs- $\mathrm{Au}$ array for 25 and $40 \mathrm{~nm}$ can be attributed to electromagnetic enhancement in hot spot region on top of the $\mathrm{ZnO}$ NRs array and their mutual interaction. The present 3D plasmonic HNS provides mechanisms of controlling charge transfer plasmon between bridged Au NPs and tuning them as functions of tunneling transport, junction conductance and NPs parameters [16].

\subsection{UV- Vis Near IR Absorption and Optical Modeling Results}

To measure the UV-visible absorption spectra of bare ZnO NRs array and ZnO NRs - Au array HNS, they were deposited on quartz substrates. The bare ZnO NRs absorption spectra are shown in Fig. 4. The absorption edge above $3 \mathrm{eV}$ is due to the interband absorption of $\mathrm{ZnO}$. The simulated absorption spectra using the lorentzian (L), Guassian imposed Forouhi- Bloomer $\left(F^{*} \mathrm{G}\right)$, Drude $(\mathrm{D})$ dispersion and finally summation of $\mathrm{L}+\mathrm{FB} * \mathrm{G}+\mathrm{Durde}$ depicted in Fig. 4. 
Theoretical absorption based on the proposed dispersion model match with the experimental absorption as well.

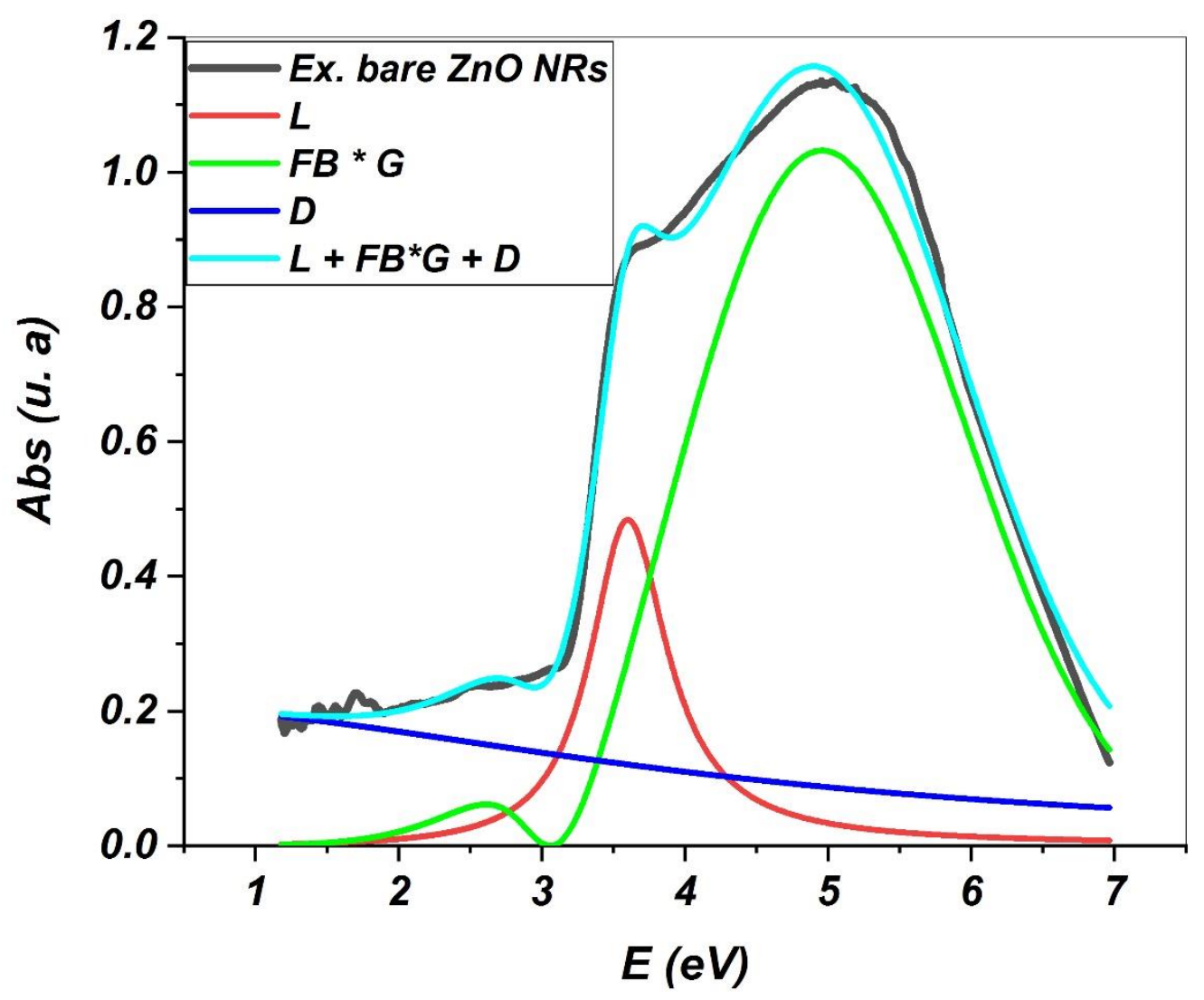

Fig. 4: Absorption spectra of the bare ZnO NRs array, Lorentzion oscillation dispersion, Guassian imposed FB dispersion $(F B * G)$, Drude (D) dispersion and the proposed model $(\mathrm{L}+\mathrm{FB} * \mathrm{G}+\mathrm{D})$

Fig. 5 exhibits the experimental and simulated absorption spectra for the bare $\mathrm{ZnO}$ NRs array and the ZnO NRs array- Au HNS for various Au thickness. Fig. 5a depicts that Guassian imposed FB dispersion $(\mathrm{FB} * \mathrm{G})$ gurantee sharp variation of absorption around fundamental bandgap edge in comparision to the individual FB dispersion. Fig. 5b- $f$ show the observed absorption spectra and the simulated results of $\mathrm{ZnO}$ NRs 
array - Au HNS for various Au thickness of 5 - 40nm. For ZnO NRs - Au array HNS, a broad absorption peak located below the bandedge is assigned to LSPR absorption of Au NPs. The proposed model results follow the experimental spectra and estimate localized SPR resonance as well.

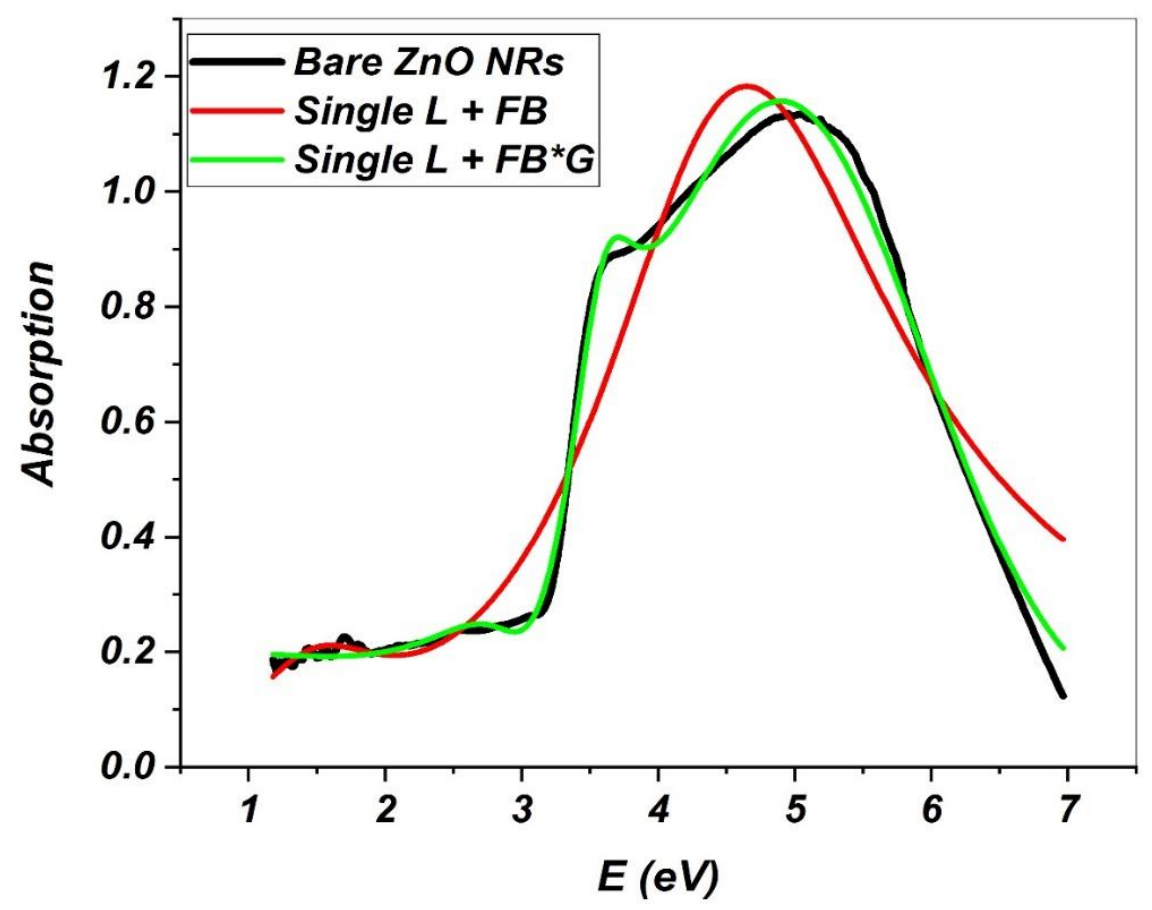



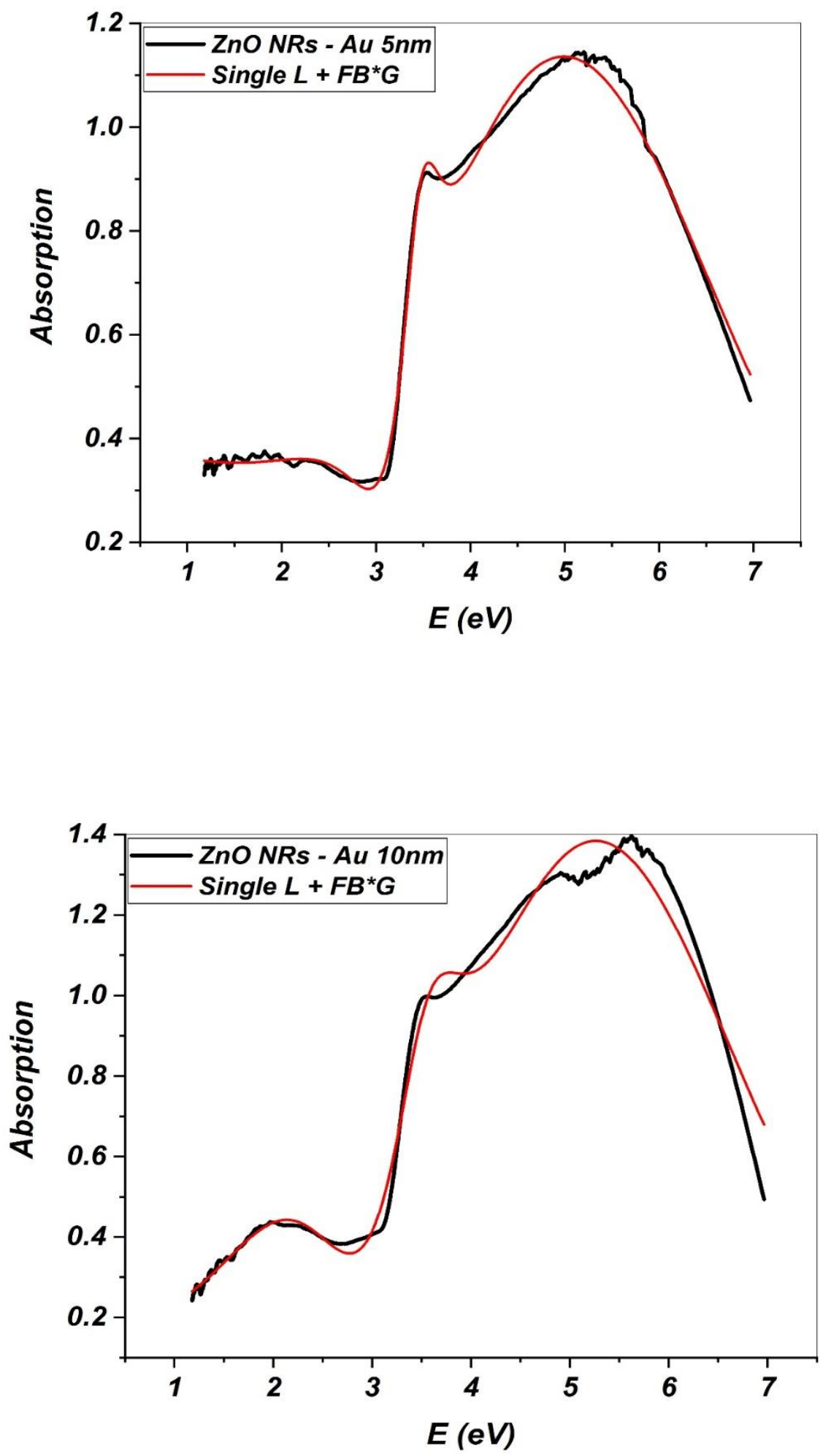






Fig.5: The observed and simulated absorption spectra of ZnO NRs array-Au HNS for different $\mathrm{Au}$ thickness of $0-40 \mathrm{~nm}$

Table 1 illustrates natural resonance energy $\left(\mathrm{E}_{0}\right)$, broadening parameter of bound electrons, threshold energy $\left(E_{t h}\right)$, bandgap energy $\left(E_{g}\right)$, band tail energy $\left(E_{\Delta}\right)$, centre and width of Guassian peak, plasmon energy $\left(E_{p}\right)$, broadening parameters of free electrons $\left(\Gamma_{D}\right)$, surface plasmon energy $\left(\mathrm{E}_{\mathrm{SPR}}\right)$ and the ration of $\mathrm{E}_{\mathrm{SPR}}$ to $\mathrm{E}_{\mathrm{p}}$. The natural resonance of $\mathrm{ZnO} \mathrm{NRs}$ array - Au hybrid weakly decreases due to hetero interface and average of dangling bound and space charge removal. $\mathrm{ZnO}-\mathrm{Au}$ hetero interface increases the damping rate of the bound electrons.

The bandgap energy overally decreases from $3.42 \mathrm{eV}$ to $3.26 \mathrm{eV}$ as Au thickness varies from 0 to $40 \mathrm{~nm}$. The absorption edge of pure $\mathrm{ZnO}$ is redshifted as $\mathrm{Au}$ thickness increases. Many body effect renormalized and shrinkaged the bandgap energy of $\mathrm{ZnO} \mathrm{NRs}$ array- $\mathrm{Au}$ in comparison to the bare $\mathrm{ZnO}$ NRs [29]. Formation of the $\mathrm{Au}$ nanosheet on $\mathrm{ZnO}$ NRs wall is evident from vaiation in optical absorption 
spectra. The position of LSPR peak is gradually blueshifted and its intensity increases systematically by increasing Au thickness. The surface plasmon (SP) absorption band of Au shifts toward higher energy due to the interaction between $\mathrm{Au}$ and $\mathrm{ZnO}$ NRs array. It is well known that small metallic particles show the optical absorption due to the SPR in the visible-near IR region. It should be noted that the broad LSPR peak is beneficial for ZnO NRs - Au array HNS to harvest the visible light as much as possible. For 5 to $15 \mathrm{~nm}$ thick Au nanosheets, the broad absorption peaks are observed in the visible region, which is blue-shifted as the thickness of $\mathrm{Au}$ increased gradually to $40 \mathrm{~nm}$. The gradual decrease in band tail energy $\left(E_{\Delta}\right)$ is evaluated in respect to renormalized band edges. The $E_{S P R}$ varies between $2.08-2.29 \mathrm{eV}$ which lies in the green region of light spectrum. It is surprising that this model covers and predicts SPR peaks without introducing special formalism.

Table 1: The estimated model parameters

\begin{tabular}{|c|c|c|c|c|c|c|c|c|c|c|c|}
\hline sample & $\begin{array}{c}\mathbf{E}_{0} \\
(\mathrm{eV})\end{array}$ & $\begin{array}{c}\Gamma_{b} \\
\left(10^{-15}\right. \\
\mathbf{H z})\end{array}$ & $\begin{array}{c}\text { Eth }_{\text {th }} \\
(\mathrm{eV})\end{array}$ & $\begin{array}{c}\mathbf{E}_{\mathbf{g}} \\
(\mathbf{e V})\end{array}$ & $\begin{array}{c}E_{\Delta} \\
(\mathrm{eV})\end{array}$ & $\begin{array}{c}\text { Guassian } \\
\text { centre }\end{array}$ & $\begin{array}{c}\text { Guassian } \\
\text { width }\end{array}$ & $\begin{array}{c}\mathbf{E}_{p} \\
(\mathbf{e V})\end{array}$ & $\begin{array}{l}\left(10-\Gamma_{D}\right. \\
\left.{ }^{15} \mathrm{~Hz}\right)\end{array}$ & $\begin{array}{l}\text { ESPR } \\
(e V)\end{array}$ & $\frac{E_{S P R}}{E_{p}}$ \\
\hline Bare $\mathrm{ZnO} \mathrm{NRs}$ & 3.60 & 0.62 & 3.06 & 3.42 & 0.36 & 4.63 & 2.48 & 0.40 & 4.03 & - & - \\
\hline ZnO NRs & 3.48 & 0.52 & 3.07 & 3.34 & 0.27 & 4 & 3.78 & 0.46 & 2.82 & - & - \\
\hline $\mathrm{ZnO}$ NRs $-\mathrm{Au} 10 \mathrm{~nm}$ & 3.57 & 1.02 & 3.11 & 3.34 & 0.23 & 4. & 3.8 & 0.30 & 2.83 & 2.08 & 6.96 \\
\hline $\mathrm{ZnONRs}-\mathrm{Au} 15 \mathrm{~nm}$ & 3.57 & 1.07 & 3.16 & 3.33 & 0.17 & 4.43 & 3.85 & 0.38 & 2.84 & 2.27 & 5.91 \\
\hline $\mathrm{ZnO} N R s-\mathrm{Au} 25 \mathrm{~nm}$ & 3.50 & 1.00 & 3.11 & 3.30 & 0.19 & 4.13 & 4.53 & 0.55 & 4.57 & 2.24 & 4.05 \\
\hline $\mathrm{ZnO}$ NRs $-\mathrm{Au} 40 \mathrm{~nm}$ & 3.46 & 1.43 & 3.10 & 3.26 & 0.16 & 4.33 & 4.49 & 0.40 & 0.91 & 2.29 & 5.62 \\
\hline
\end{tabular}

Table 2 shows the refractive index, high frequency and low (static) dielectric constants, bandgap energy and effective mass. The Dimitrov-Sakka relation predicts moderate value between that of Moss and Herve - Vandamme. $\varepsilon_{\infty}$ increases as $\mathrm{Au}$ thickness increases while $\varepsilon_{0}, \mathrm{E}_{\mathrm{g}}$ and $\frac{m^{*}}{m_{0}}$ shows decreasing terend versus increase in Au thickness.

Table 2: refractive index, high frequency and static dielectric constant, bandgap energy and effective mass 


\begin{tabular}{|c|c|c|c|c|c|c|c|c|}
\hline \multirow[b]{2}{*}{ sample } & \multicolumn{3}{|c|}{$\mathbf{n}$} & \multicolumn{3}{|c|}{$\varepsilon_{\infty}$} & \multirow[b]{2}{*}{$\varepsilon_{0}$} & \multirow[b]{2}{*}{$\mathbf{m}^{*} / \mathbf{m}_{\mathbf{0}}$} \\
\hline & Moss & $\begin{array}{c}\text { Herve- } \\
\text { Vandamme }\end{array}$ & $\begin{array}{c}\text { Dimitrov- } \\
\text { Sakka }\end{array}$ & Moss & $\begin{array}{c}\text { Herve- } \\
\text { Vandamme }\end{array}$ & $\begin{array}{l}\text { Dimitrov- } \\
\text { Sakka }\end{array}$ & & \\
\hline Bare $\mathrm{ZnO}$ NRs & 2.37 & 2.23 & 2.29 & 5.62 & 4.98 & 5.25 & 33.98 & 0.89 \\
\hline $\mathrm{ZnO} \mathrm{NRs} \mathrm{-} \mathrm{Au} 5 \mathrm{~nm}$ & 2.38 & 2.25 & 2.31 & 5.69 & 5.07 & 5.34 & 29.58 & 0.68 \\
\hline $\mathrm{ZnO}$ NRs $-\mathrm{Au} 10 \mathrm{~nm}$ & 2.38 & 2.25 & 2.31 & 5.69 & 5.07 & 5.34 & 29.58 & 0.68 \\
\hline $\mathrm{ZnO} \mathrm{NRs}-\mathrm{Au} 15 \mathrm{~nm}$ & 2.39 & 2.25 & 2.31 & 5.69 & 5.08 & 5.35 & 29.07 & 0.65 \\
\hline $\mathrm{ZnO}$ NRs $-\mathrm{Au} 25 \mathrm{~nm}$ & 2.39 & 2.26 & 2.32 & 5.72 & 5.12 & 5.38 & 27.57 & 0.58 \\
\hline $\mathrm{ZnO} \mathrm{NRs}-\mathrm{Au} 40 \mathrm{~nm}$ & 2.40 & 2.27 & 2.33 & 5.76 & 5.16 & 5.43 & 25.67 & 0.50 \\
\hline
\end{tabular}

\subsection{Exemplification of the constructed model: $\mathrm{CuO}$ thin film}

To test and generalize the constructed model, $\mathrm{CuO}$ thin film of $100 \mathrm{~nm}$ thickness has been evaluated. It is seen that the model result is well coincide with experimental data (Fig. 5). The predicted bandgap energy is $1.98 \mathrm{eV}$ [21]. Also the model meet good fitting below and above band to band transition.

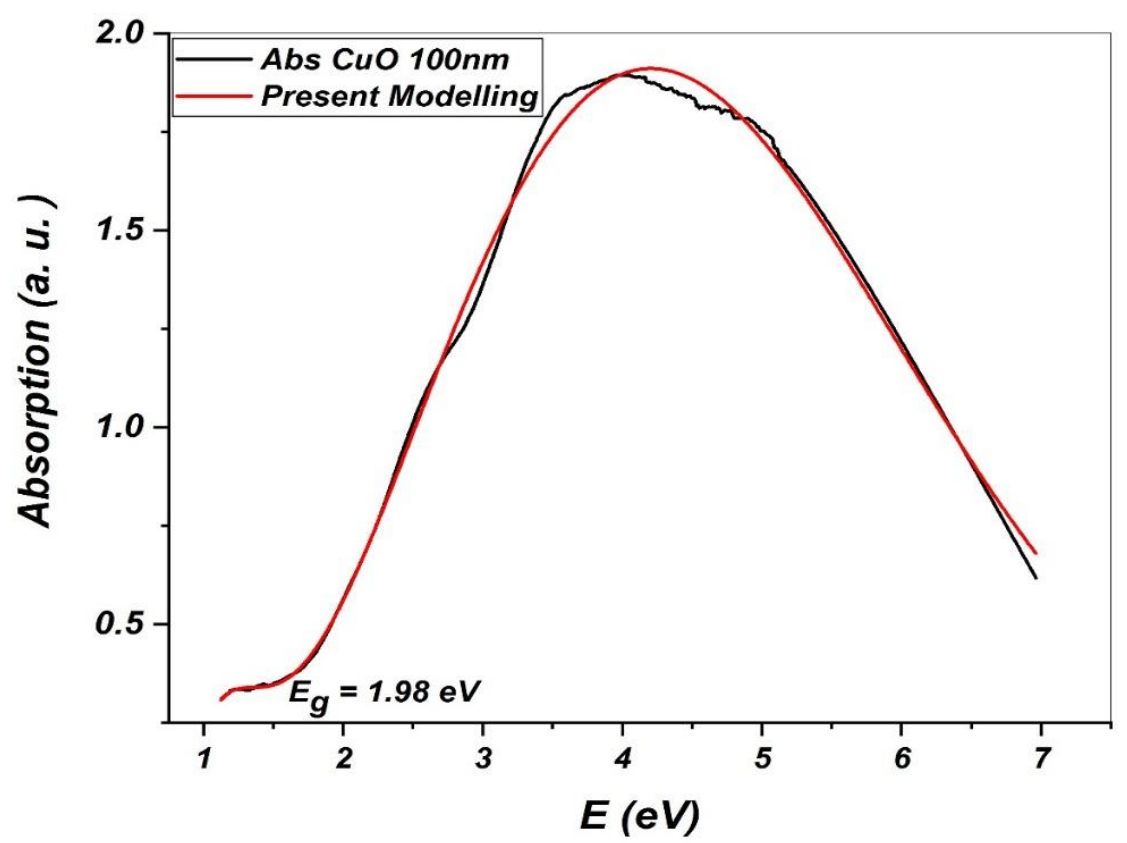

Fig 5: $\mathrm{CuO}$ absorption spectra and present modeling

\subsection{Photogain study}


To evaluate the light sensitivity of $\mathrm{ZnO}$ NRs- $\mathrm{Au}$ 40nm array HNS, the photogain (photocurrent density per light power) was examined. Fig. 8 depicts the photogain - voltage characteristic of ZnO NRs- Au 40nm array HNS under the illumination of red, green and blue laser versus sweep voltage. The photosensitivity has higher value for green illumination in comparison to the red and blue light. SPR excitation in green region of electromagnetic spectrum spoil the carriers and surface charge transfer [16].

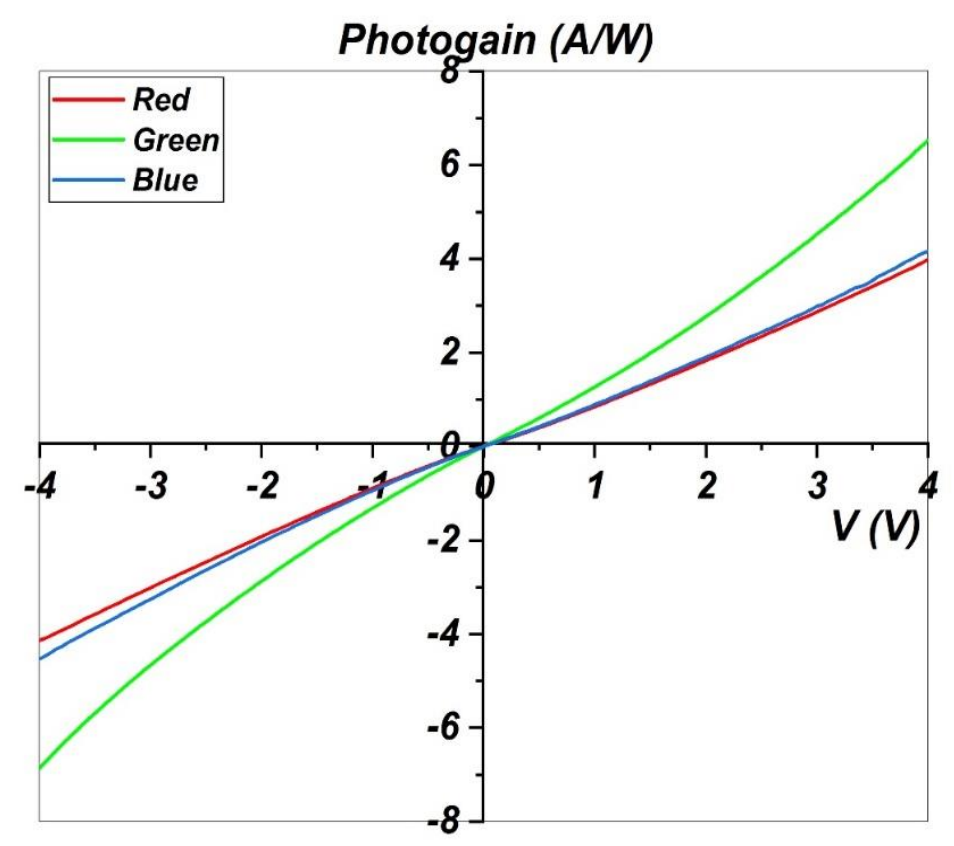

Fig6: Photogain - voltage characteristic of $\mathrm{ZnO}$ NRs- $\mathrm{Au} 40 \mathrm{~nm}$ array HNS under the illumination of red, green and blue laser illumination.

The higher green photoresponse of $\mathrm{ZnO}$ NRs-Au HNSs mainly results from the hot electron generation and transfer process over the Schottky junctions mediated by Au SPR. The Au nanosheet acts as an electron source which spill electrons into $\mathrm{ZnO}$ conduction band due to SP 
excitation in ZnO NRs- Au array HNS. Charge transfer plasmons inject excess electrons from $\mathrm{Au}$ SP band to $\mathrm{ZnO}$ NRs.

\section{Conclusion}

A three-dimensional (3D) plasmonic ZnO NRs- Au array hetero-nanostructure (HNs) was grown using wet chemical method and magnetron sputtering deposition at oblique angle to improve visible light induced photoactive properties. The surface plasmon excitation play important role to charge transfer plasmon and selective green response. Forouhi- Bloomer dispersion is modified by Guassian dispersion, well follows experimental trend beyond the bandgap edge and guarantees sharp variation around the bandgap edge. It is surprising that the proposed model predicts surface plasmon resonance without introducing individual formalisms.

Funding: Not applicable

Conflicts of interest/Competing interests: This is our research paper. Without the participation of anyone and without oppressing anyone. We are fully responsible for it. Availability of data and material: Not applicable

Code availability: Not applicable

Authors' contributions: Not applicable

Ethics approval: Not applicable

Consent to participate: Not applicable

Consent for publication: Not applicable

\section{References}

[1] K. Zhang, A. R. Forouhi, and I. Bloomer Accurate and rapid determination of thickness, $\mathrm{n}$ and $\mathrm{k}$ spectra, and resistivity of indium-tin-oxide films, J. Vac. Sci. Technol. A 17.4., 1999, 1843- 1847 [2] Zhen Li, Shun Hui Lin, Gui Ming Qiu, Jiang Yong Wang, and Yun Peng Yu A method for determining band parameters from the optical absorption edge of amorphous semiconductor: Application to a-Si:H, JOURNAL OF APPLIED PHYSICS 124, 025702 (2018) 
[3] D. Franta, M. Cermak, J. Vohanka and I. Ohlidal, Dispersion models describing interband electronic transitions combining Tauc's law and Lorentz model, Thin Solid Films 631 (2017) 12-22

[4] D.V. Likhachev, N.Malkova , L. Poslavsky, Modified Tauc-Lorentz dispersion model leading to a more accurate representation of absorption features below the bandgap, Thin Solid Films 589 (2015) 844-851

5. D. Campi and C. Coriasso, "Prediction of optical properties of amorphous tetrahedrally bonded materials," J. Appl. Phys. 64(8), 4128-4134 (1988).

6. G. E. Jellison and F. A. Modine, "Parameterization of the optical functions of amorphous materials in the interband region," Appl. Phys. Lett. 69(3), 371-373 (1996).

[7] G. D. Cody, Semiconductors and Semimetals, vol. 21 (Academic, 1984).

[ 8] L. V. RODRÍGUEZ-DE MARCOS AND J. I. LARRUQUERT Analytic optical-constant model derived fromTauc-Lorentz and Urbach tail , 24. 25 OPTICS EXPRESS 2856

[9] M. Foldyna, K. Postava, J. Bouchala, J. Pitora, and T. Yamaguchi, "Model dielectric function of amorphous materials including Urbach tail," Proc. SPIE 5445, 301-305 (2003).

[10] A. S. Ferlauto, J. Koh, P. I. Rovira, C. R. Wronski, R. W. Collins, and G. Ganguly, "Modeling the dielectric functions of silicon-based films in the amorphous, nanocrystalline and microcrystalline regimes," J. Non-Cryst. Solids 266-269, 269-273 (2000).

[11] D. Franta, I. Ohlídal, D. Nečas, F. Vižd'a, O. Caha, M. Hasoň, P. Pokorný, Optical characterization of HfO2 thin films, Thin Solid Films 519 (2011) 6085-6091.

[12] A.S. Ferlauto, G.M. Ferreira, J.M. Pearce, C.R. Wronski, R.W. Collins, X. Deng, G. Ganguly, Analytical model for the optical functions of amorphous semiconductors and its applications for thin film solar cells, Thin Solid Films 455-456 (2004) 388-392.

[13] J.J. Mei, H. Chen, W.Z. Shen, H.F.W. Dekkers, Optical properties and local bonding configurations of hydrogenated amorphous silicon nitride thin films, J. Appl. Phys. 100 (2006) 073516.

[14] N Shahi, A Rahmati, Ag Incorporated ZnO Nanorods Array/ZnSe Heterostructure, ECS Journal of Solid State Science and Technology 8(10) (2019) Q200-Q206

[15] A Rahmati, A Farokhipour, Rectifying Behaviour and Photocatalytic Activity in ZnO Nanorods Array/ Ag /

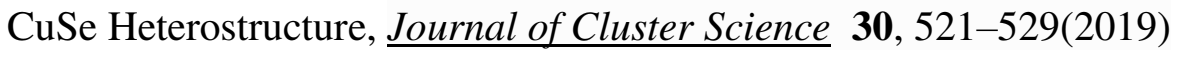


[16]M. Ghaemi-moghadam , A. Hasanzadeh , A. Rahmati Charge transfer plasmon coupled surface photosensing in $\mathrm{ZnO}$ nanorods-Au array hetero-nanostructures, Optics and Lasers in Engineering 137 (2021) 106384

[17] M. FOX, Optical Properties of Solids, Second Edition 2010, Oxford University Press, page 39

[18] H. Fujiwara and M. Kondo, Effects of carrier concentration on the dielectric function of $\mathrm{ZnO}: \mathrm{Ga}$ and $\mathrm{In}_{2} \mathrm{O}_{3}$ :Sn studied by spectroscopic ellipsometry: Analysis of free-carrier and bandedge absorption, PHYSICAL REVIEW B 71, 0751092005

[19] D. De Sousa Meneses, M. Malki, P. Echegut, Structure and lattice dynamics of binary lead silicate glasses investigated by infrared spectroscopy, J. Non-Cryst. Solids 352 (2006) 769-776.

[20] T. Easwarakhanthan, D. Beyssen, L. Le Brizoual, and P. Alnot, Forouhi-Bloomer and TaucLorentz optical dispersions applied using spectroscopic ellipsometry to plasma-deposited fluorocarbon films, Journal of Applied Physics 101, 073102 (2007)

[21] Y. Akaltun, M.A. Yıldırım, A. Ateş, M. Yıldırım, Zinc concentration effect on structural, optical and electrical properties of Cd1-xZnxSe thin films. Mater.Ress. Bull. 47 (2012) 3390- 3396.

[22] F. Mezrag, W.K. Mohamed, N. Bouarissa, The effect of zinc concentration upon optical and dielectric properties of $\mathrm{Cd}_{1-\mathrm{x}} \mathrm{Zn}_{\mathrm{x}} \mathrm{Se}$. Physica B. 405 (2010) 22722276.

[23] P. Herve, L.K.J. Vandamme, General relation between refractive index and energy gap in semiconductors. Infrared Phys. Technol. 35 (1994) 609-615

[24] V. Dimitrov, S. Sakka, Linear and nonlinear optical properties of simple oxides. II, J. Appl. Phys. 79 (3), 1 February 1996, 1741 - 1745

[25] L. Hannachi, N. Bouarissa, Band parameters for cadmium and zinc chalcogenide compounds. Physica B. 404 (2009) 3650-3654. 
[26] V Gupta, P. Bhattacharya, Y I. Yuzuk, K. Sreenivas, R.S. Katiyar, Optical phonon modes in $\mathrm{ZnO}$ nanorods on Si prepared by pulsed laser deposition, Journal of Crystal Growth 287 (2006) $39-43$

[27] K. Alim, V.A. Fonoberov, M. Shamsa and A.A. Balandin, "Micro-Raman investigation of optical phonons in ZnO quantum dots," J. Appl. Phys. 97, 124313 (2005).

[28]D Choi, Y Choi, S Hong, T Kang, and L P. Lee, Self-Organized Hexagonal-Nanopore SERS Array, DOI: 10.1002/smll.200901937, small 2010, 6, No. 16, 1741-1744

[29 ] A Rahmati, B Rahmani, A Farokhipour, Hetero plasmonic 2D and 3D ZnO/Ag nanostructures: electrical and photocatalytic applications. Journal of Materials Science: Materials in Electronic 29 (2018) 6350- 6360 